\title{
Differential transformation method for solving sixth-order boundary value problems of ordinary differential equations
}

\begin{abstract}
In this study, sixth-order boundary value problems for linear and nonlinear differential equations have been solved by using Differential Transformation Method (DTM). The numerical solutions are given in several examples. For each example, the solution given by DTM is compared with the exact solution. Absolute relative error (ARE) for each iteration can be computed. Therefore, the maximum absolute relative error (MARE) of the DTM can be obtained. To show that the solution given by the DTM has higher level of accuracy, the absolute relative error of the DTM has been compared with the other methods such as Adomian decomposition method with Green's function, modified decomposition method (MDM), homotopy perturbation method (HPM), Variational Iteration Method (VIM) and Quintic B-Spline Collocation Method. Comparison graphs are given at the end of this paper. The obtained result shows that the proposed method is able to provide better approximation in term of accuracy.
\end{abstract}

Keyword: Absolute error; Differential transformation method; Ordinary differential equations,; Series solution; Sixth-order boundary value problems 